\title{
Study on the 3D anisotropic propagation of Galactic cosmic rays
}

\author{
Wei Liu ${ }^{a}$, Su-Jie Lin ${ }^{a}$, Hong-Bo Hu ${ }^{a b}$, Yi-Qing Guo ${ }^{a}$ \\ ${ }^{a}$ Institute of High Energy Physics, Chinese Academy of Sciences \\ Beijing 100049, China \\ ${ }^{b}$ University of Chinese Academy of Sciences, Beijing 100049, China E-mail: \\ liuwei@ihep.ac.cn, linsjeihep.ac.cn, huhb@ihep.ac.cn, \\ guoyqdihep.ac.cn
}

Conventional cosmic-ray propagation models usually assume an isotropic diffusion coefficient to account for the random deflection of cosmic rays by the turbulent interstellar magnetic field. Such a picture is very successful in explaining a lot of observational phenomena related to the propagation of galactic cosmic rays, such as broken power-law energy spectra, secondary-to-primary ratios, etc. However, the isotropic diffusion presupposition is facing severe challenges from observations. In particular, recent observations on the large-scale anisotropy of $\mathrm{TeV}$ cosmic rays show that the dipole direction differs from the prediction of the conventional model. One possible reason is that the large-scale regular magnetic field, which leads to an anisotropic diffusion of cosmic rays, has not been included in the model provided by the public numerical packages. In this work, we propose a pseudo source method to solve the 3-dimensional anisotropic transport equation. Based on a realistic configuration of Galactic magnetic field, both proton energy spectrum and $\mathrm{B} / \mathrm{C}$ ratio are reproduced with proper transport parameters.

36th International Cosmic Ray Conference -ICRC2019-

July 24th - August 1st, 2019

Madison, WI, U.S.A. 


\section{Introduction}

It has long been recognized that after escaping from the acceleration sites, the Galactic cosmic rays (GCRs) undergo frequent scatterings with the random magnetic turbulence, which could be phenomenologically described by a diffusion process. In the conventional model, the diffusion process is supposed to be uniform and isotropic, in which the diffusion coefficient is only rigiditydependent, namely $D(\mathscr{R}) \propto \mathscr{R}^{\delta}$, with $\delta \sim 0.3-0.6$ inferred from the boron-to-carbon ratio [1]. The propagated CR spectrum falls off as $\phi \propto \mathscr{R}^{-v-\delta}$, where $v$ is an injection power index. This CR transport picture has successfully reproduced some basic observational features, for example, the power-law form of the energy spectra, the secondary-to-primary ratio, and the large-scale distribution of diffuse radio/gamma-ray radiation.

In the past decades, the high-precision and two dimensional observations of CR anisotropy $[2,3,4,5,6,7,8,9,10,11,12,13,14,15,16]$ disfavor such a simple picture. Both the amplitude and phase could not be reproduced by the conventional model. Based on the conventional anisotropic propagation model, various efforts have been explored to explain the anisotropy problem. These include ensemble fluctuations of CR sources, nearby source $[17,18,19,20]$ and spatial dependent diffusion process $[21,22,23]$ etc. Local effects are usually sensitive to both the amplitude and phase of the anisotropy, the spatial dependent diffusion coefficient is mainly sensitive to the amplitude.

On the other hand, the local regular magnetic field has been introduce to understand the phase of CR anisotropy. The studies of [24, 25, 26] propose that the local regular magnetic field may have a projection effect to $\mathrm{CR}$ streaming. These works attribute the multi-TeV energy anisotropy to the contribution from nearby SNRs. The local CRs are assumed to propagate to the solar system along the regular magnetic field line. Both amplitude and phase of anisotropy could be phenomenologically described in the view of the anisotropic propagation of GCRs. For the purpose of accurate calculation, it is necessary to implement the anisotropic propagation in the public numerical packages, such as GALPROP and DRAGON.

In this work, we propose a pseudo source method to solve the anisotropic propagation equation. Demonstrated by a toy magnetic field model, we verify the validity of the iterative method and find that the method is able to converge only after a few iterations. We further apply our numerical package to fit the proton energy spectrum and $\mathrm{B} / \mathrm{C}$ ratio, under a realistic magnetic field model, and obtain the corresponding diffusion parameters.

The rest of paper is organized as follows: In Sec. 2, anisotropic diffusion equation and the corresponding iteration method are introduced. The realistic regular magnetic field configuration is also described in detail. Sec. 3 presents the calculation results using two magnetic field models and compares the density distribution, the proton spectrum and $\mathrm{B} / \mathrm{C}$ ratio with relevant calculations or observations. Sec. 4 is reserved for the conclusion.

\section{Method}

As a matter of fact, the anisotropic diffusion is available for the GALPROP and DRAGON packages, but only in a few special cases. One is allowed to use different diffusion coefficients only for the given coordinate axis, but not for the general situation. 


\subsection{Anisotropic diffusion}

A comprehensive introduction to GCR production and propagation could be found in many monographs and review papers, such as [27, 28, 29, 30]. Here we give a brief review on GCR propagation. The diffusive region of GCRs, also called magnetic halo, is usually approximated as a cylinder, with its radial boundary equal to the Galactic radius, i.e. $R=20 \mathrm{kpc}$. Its half thickness $L$, which characterizes the vertical stretch of interstellar magnetic field, is a free parameter, which is determined by fitting the $\mathrm{B} / \mathrm{C}$ ratio. Both $\mathrm{CR}$ sources and interstellar medium (ISM) are chiefly distributed in the Galactic disk with average thickness $z_{s}$ of roughly $200 \mathrm{pc}$. The transport process of CRs in the magnetic halo is described by the following convection-diffusion equation

$$
\begin{aligned}
\frac{\partial \psi(\vec{r}, p, t)}{\partial t} & =q(\vec{r}, p, t)+\nabla \cdot(D \nabla \psi)-\nabla \cdot\left(\vec{V}_{c} \psi\right) \\
& +\frac{\partial}{\partial p} p^{2} D_{p p} \frac{\partial}{\partial p} \frac{1}{p^{2}} \psi-\frac{\partial}{\partial p}\left[\dot{p} \psi-\frac{p}{3}\left(\nabla \cdot V_{c} \psi\right)\right] \\
& -\frac{\psi}{\tau_{f}}-\frac{\psi}{\tau_{r}} .
\end{aligned}
$$

Here $\psi(\vec{r}, p, t)$ is the CR density per unit energy at time $t$. The terms containing $\vec{V}_{c}, D_{p p}, \dot{p}, \tau_{f}$ and $\tau_{r}$ describe the convection, diffusive re-acceleration, energy-loss, fragmentation and radiative decay effects correspondently. $D$ is so-called spatial diffusion coefficient, which is in general expressed as a rank two symmetric tensor, and thus the diffusion term is written as

$$
\nabla \cdot(D \nabla) \psi=\frac{\partial}{\partial x_{i}}\left(D_{i j} \frac{\partial \psi}{\partial x_{j}}\right)
$$

For example, for the two optional coordinate systems in the GALPROP and DRAGON packages, $\left(x_{1}, x_{2}\right)$ denotes $(r, z)$ and $\left(x_{1}, x_{2}, x_{3}\right)$ denotes $(x, y, z)$ in the cylinder and Cartesian coordinate systems respectively. When $D_{i j}=D \delta_{i j}$, the diffusion is isotropic, otherwise, it is anisotropic. As mentioned previously, both GALPROP and DRAGON packages can deal with the anisotropic diffusion if the off-diagonal terms become zero.

The ordered magnetic field plays important role in the anisotropic diffusion. By numerical calculation, the trajectory of a charged particle can be precisely traced in an environment filled with both the regular and irregular magnetic field [31]. According to these studies, an anisotropic diffusion coefficient become necessary when the regular magnetic field strength is comparable to or beyond that of the turbulent one. The diffusion coefficient tensor can be diagonalized at every point by properly choosing the local coordinate, with one axis parallel and other two perpendicular to the direction of local regular magnetic field. In this local coordinate system, the diffusion tensor looks like

$$
D_{i j}=\left(\begin{array}{ccc}
D_{\|} & 0 & 0 \\
0 & D_{\perp} & 0 \\
0 & 0 & D_{\perp}
\end{array}\right) .
$$

Here $D_{\|}$and $D_{\perp}$ are the diffusion coefficients aligned with and perpendicular to the ordered magnetic field respectively. Practically numerical calculation has to be performed in one global coordinate system, therefore the non-zero off-diagonal terms is inevitable. When transforming the above local coordinate system defined by the regular magnetic field to the global one, $D_{i j}$ turns to be [32]

$$
D_{i j} \equiv D_{\perp} \delta_{i j}+\left(D_{\|}-D_{\perp}\right) b_{i} b_{j},
$$


where $b_{i}=\frac{B_{i}}{|\vec{B}|}$ is the $i$-th component of the unit vector of the ordered magnetic field $\vec{B}$ in a chosen coordinate system. Following the work [33], $D_{\|}$and $D_{\perp}$ have different rigidity dependence

$$
\begin{aligned}
& D_{\|}=D_{0 \|}\left(\frac{\mathscr{R}}{\mathscr{R}_{0}}\right)^{\delta_{\|}} \\
& D_{\perp}=D_{0 \perp}\left(\frac{\mathscr{R}}{\mathscr{R}_{0}}\right)^{\delta_{\perp}} \equiv \varepsilon D_{0 \|}\left(\frac{\mathscr{R}}{\mathscr{R}_{0}}\right)^{\delta_{\perp}}
\end{aligned}
$$

in which $\varepsilon=\frac{D_{0 \perp}}{D_{0 \|}}$ is the ratio between perpendicular and parallel diffusion coefficient at reference rigidity $\mathscr{R}_{0}$.

\subsection{Pseudo source solution to off-diagonal diffusion terms}

To accommodate for the non zero off-diagonal terms of diffusion tensor in the current numerical package, an ideal way is to include these term implicitly in the calculation. However, such a solution for 3 dimension propagation does not exist so far. In this work, we propose a practical treatment by moving these additional diffusion terms into the source term, based on GALPROP numerical package. In another words, the first two terms of rhs. of equation 2.1 become

$$
\begin{aligned}
& q(\vec{r}, p, t)+\nabla \cdot(D \nabla \psi) \\
& =\left[q(\vec{r}, p, t)+\sum_{i \neq j} \frac{\partial}{\partial x_{i}}\left(D_{i j} \frac{\partial \psi}{\partial x_{j}}\right)\right]+\frac{\partial}{\partial x_{i}}\left(D_{i i} \frac{\partial \psi}{\partial x_{i}}\right) \\
& =\left[q(\vec{r}, p, t)+q_{\text {pseudo }}(\vec{r}, p, t)\right]+\frac{\partial}{\partial x_{i}}\left(D_{i i} \frac{\partial \psi}{\partial x_{i}}\right) \\
& =q^{\prime}(\vec{r}, p, t)+\frac{\partial}{\partial x_{i}}\left(D_{i i} \frac{\partial \psi}{\partial x_{i}}\right)
\end{aligned}
$$

where $q_{\text {pseudo }}$ is called the pseudo source, which represents the off-diagonal terms of diffusion tensor. $q^{\prime}$ is the sum of real and pseudo sources. In such a way, the propagation equation with $3 \mathrm{D}$ off-diagonal terms returns to the original form, which is perfectly fit for the GALPROP package.

The iteration method is used to solve this equation. In the first step, no pseudo source is assumed. We just solve a diffusion equation by neglecting off-diagonal terms and obtain a firstorder CR distribution $\psi^{(1)}$. After the first iteration, the pseudo source $q_{\text {pseudo }}$ can be constructed according to the definition in Equ. (2.6), by using the CR distribution obtained from the last iteration. After solving this equation, a second-order distribution $\psi^{(2)}$ is obtained. Repeating such process until the solution comes to a converge, the final distribution is obtained.

\subsection{Verification with a toy magnetic field model}

Before applying the realistic magnetic field configuration, we adopt a 2D toy model used by [34] and compare our method with their 2D anisotropic diffusion calculation. The toy model is 
described as

$$
\begin{aligned}
& B_{r}=0 \\
& B_{\phi}=B_{0, \phi}\left(1-\exp \left[-\frac{r}{r_{0}}\right]\right) \\
& B_{z}=B_{0, z} \exp \left[-\frac{r}{r_{0}}\right] \equiv \varepsilon_{B} B_{0, \phi} \exp \left[-\frac{r}{r_{0}}\right]
\end{aligned}
$$

in which $\varepsilon_{B}=\frac{B_{0, z}}{B_{0, \phi}}$.

This simple toy model could roughly describe the Galactic magnetic field. Close to the Galactic center, $B_{z}$ is the major component of magnetic field, but $B_{\phi}$ is dominated over $B_{z}$ far away from the Galactic center. Therefore with increasing distance, the CR diffusion gradually changes from parallel diffusion dominated (near the Galactic center) to the perpendicular diffusion dominated (at large distance). So a radial variation of the spectral index of the propagated spectrum is expected if $\delta_{\|} \neq \delta_{\perp}$, i.e.

$$
\psi(\mathscr{R})= \begin{cases}\mathscr{R}^{-\left(\alpha+\delta_{\|}\right)}, & r \ll R_{0} \\ \mathscr{R}^{-\left(\alpha+\delta_{\perp}\right)}, & r \gg R_{0}\end{cases}
$$

In Fig. 1, we show the radial variation of the spectral index of proton computed by our iteration method. The red, blue and green points represent $\varepsilon=0.01,0.1$ and 1 respectively, while the circle and square indicate $z_{h}=2$ and $4 \mathrm{kpc}$. Here $\delta_{\|}=0.1, \delta_{\perp}=0.6, R_{0}=3.5 \mathrm{kpc}$, and $\varepsilon_{B}=0.2$. It could be seen that the spectral index gradually grows with increasing distance.

\section{Results}

To compare with the observational data, a realistic distribution of magnetic field must be used.

\subsection{Regular large scale structure of Galactic magnetic field}

Abundant measurements indicate that the Galactic magnetic field has a large-scale regular field, which has a comparable magnitude as the turbulent component [35]. The observations to other spiral galaxies also arrive at similar conclusions [36, 37]. As for our Galaxy, the regular magnetic field contains three components: 1) the disk components are along spiral arms, with counter clockwise direction in all inner arms when viewed from the northern galactic pole, evidences indicate that in the inter arm region, the magnetic field is in clockwise direction. 2) Antisymmetric azimuthal halo or toroidal magnetic field above and below the galactic plane. 3) Poloidal or Xshape halo magnetic fields cross over the galactic plane. It is nearly perpendicular to the galactic plane when close to the galactic center but has a tilt angle when it is away from the galactic center. In this work, a realistic magnetic field model is adopted according to [38, 39]. The detailed form of magnetic field is described as follows.

The disk component is taken as azimuthally symmetric, which is a function of $r$ and $z$, i.e.

$$
B_{\phi}^{\mathrm{disk}}(r, z)=\left\{\begin{array}{cc}
B_{0}^{\mathrm{d}} \exp \left(-\frac{|z|}{z_{0}}\right), & r \leqslant R_{c}^{\mathrm{d}} \\
B_{0}^{\mathrm{d}} \exp \left(-\frac{\left(r-r_{\odot}\right)}{R_{0}}-\frac{|z|}{z_{0}}\right), & r>R_{c}^{\mathrm{d}}
\end{array},\right.
$$




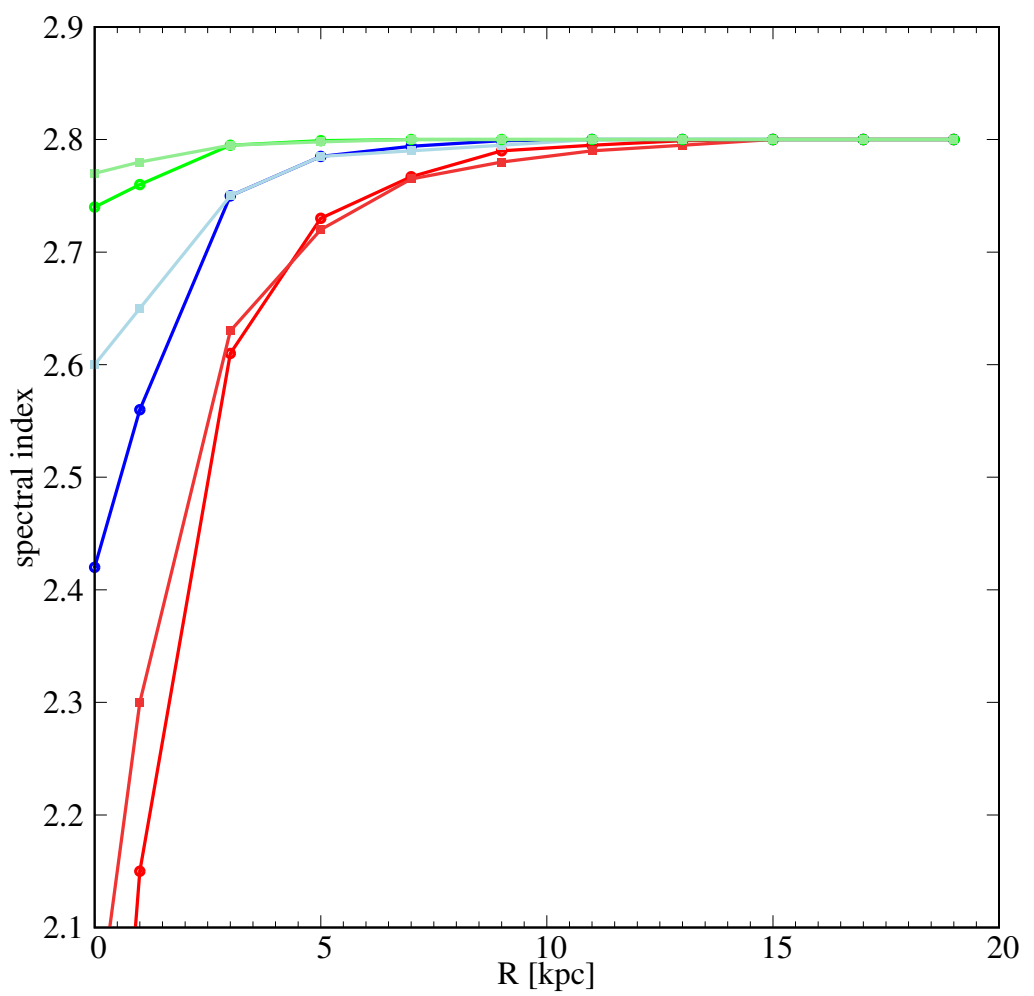

Figure 1: Radial variation of spectral index of proton spectrum with $\varepsilon=0.01$ (red), $\varepsilon=0.1$ blue and $\varepsilon=1$ (green) respectively. The circle and square points indicate $z_{h}=2$ and $4 \mathrm{kpc}$. Here $\delta_{\|}=0.1, \delta_{\perp}=0.6$, $R_{0}=3.5 \mathrm{kpc}$, and $\varepsilon_{B}=0.2$.

The azimuthal halo component is parameterized as

$$
B_{\phi}^{\text {halo }}(r, z)=B_{0}^{\mathrm{h}}\left[1+\left(\frac{|z|-z_{0}^{\mathrm{h}}}{z_{1}^{\mathrm{h}}}\right)\right]^{-1} \frac{r}{R_{0}^{\mathrm{h}}} \exp \left(1-\frac{r}{R_{0}^{\mathrm{h}}}\right) .
$$

The direction of the magnetic field in the north hemisphere is along counter-clockwise direction, while it is reversed in the southern part.

The halo poloidal magnetic field adopted here is the parametrized as,

$$
\begin{aligned}
& B^{\mathrm{pol}}(r, z)=B_{\mathrm{X}}(r, z) \exp \left(-\frac{R^{\mathrm{p}}}{R^{\mathrm{X}}}\right) \cos \left[\Theta_{\mathrm{X}}(r, z)\right], \\
& B_{r}^{\mathrm{pol}}(r, z)=B_{\mathrm{X}}(r, z) \exp \left(-\frac{R^{\mathrm{p}}}{R^{\mathrm{X}}}\right) \sin \left[\Theta_{\mathrm{X}}(r, z)\right],
\end{aligned}
$$

with $B_{\mathrm{X}}, \Theta_{\mathrm{X}}$ and $R^{\mathrm{p}}$ defined as

$$
B_{\mathrm{X}}(R, z)=\left\{\begin{array}{c}
B_{0}^{\mathrm{X}}\left(\frac{R_{p}}{r}\right)^{2}, r \leqslant R_{c}^{\mathrm{X}} \\
B_{0}^{\mathrm{X}}\left(\frac{R_{p}}{r}\right), r>R_{c}^{\mathrm{X}}
\end{array},\right.
$$




\begin{tabular}{ccccc}
\hline \hline Disk & $B_{0}^{\mathrm{d}}[\mu \mathrm{G}]$ & $z_{0}[\mathrm{kpc}]$ & $R_{c}^{\mathrm{d}}[\mathrm{kpc}]$ & \\
& 2.0 & 1.0 & 5.0 & \\
\hline Halo & $B_{0}^{\mathrm{h}}[\mu \mathrm{G}]$ & $z_{0}^{\mathrm{h}}[\mathrm{kpc}]$ & $z_{1}^{\mathrm{h}}[\mathrm{kpc}]$ & $R_{0}^{\mathrm{h}}[\mathrm{kpc}]$ \\
& 4 & 1.3 & 0.25 & 8 \\
\hline Poloidal & $B_{0}^{\mathrm{X}}[\mu \mathrm{G}]$ & $\Theta_{0}^{\mathrm{X}}$ & $R_{c}^{\mathrm{X}}[\mathrm{kpc}]$ & $R^{\mathrm{X}}[\mathrm{kpc}]$ \\
& 4.6 & $49^{\circ}$ & 4.8 & 2.9 \\
\hline
\end{tabular}

Table 1: Parameters of Galactic magnetic field model.

$$
\Theta_{\mathrm{X}}(r, z)=\left\{\begin{array}{cc}
\tan ^{-1}\left(\frac{|z|}{r-R^{\mathrm{p}}}\right), & r \leqslant R_{c}^{\mathrm{X}} \\
\Theta_{0}^{\mathrm{X}}, & r>R_{c}^{\mathrm{X}}
\end{array},\right.
$$

and

$$
R^{\mathrm{p}}=\left\{\begin{array}{cl}
\frac{r R_{c}^{\mathrm{X}}}{R_{c}^{\mathrm{X}}+\frac{|z|}{\tan \Theta_{0}^{\mathrm{X}}}}, r \leqslant R_{c}^{\mathrm{X}} \\
r-\frac{|z|}{\tan \Theta_{0}^{\mathrm{X}}}, \quad r>R_{c}^{\mathrm{X}}
\end{array}\right.
$$

All of the parameters are taken from $[38,39]$ and listed in the table 1 .

\subsection{B/C ratio and Proton spectrum}

To study the effect of 3D anisotropic diffusion with above mentioned magnetic field model, the proton spectrum and $\mathrm{B} / \mathrm{C}$ ratio are calculated with a plain diffusion model without including the convection and diffusive re-acceleration terms.

Fig. 2 illustrates the fitting to B/C ratio with different sets of parameters. The propagation and injection parameters are listed in the table 2 and ?? respectively. As shown in the table, when $\varepsilon$ decreasing, both $D_{0 \|}$ and $\delta_{\perp}$ gradually increase. In the isotropic diffusion, the $\mathrm{B} / \mathrm{C}$ ratio is proportional to $L / D$. Nearby the solar system, the diffusion is dominated by the perpendicular diffusion, i.e. $D_{\perp}$. Thus $D_{\perp}$ is determined by the $\mathrm{B} / \mathrm{C}$ ratio, when $L$ is fixed. Then as the ratio of perpendicular to parallel diffusion $\varepsilon$ drops, $D_{0 \|}$ has to rise. Under all three cases, the calculated $\mathrm{B} / \mathrm{C}$ ratio can fit the observations.

With the propagated parameters determined by fitting $\mathrm{B} / \mathrm{C}$ ratio, the propagated proton spectrum can be obtained. Fig. 3 shows the propagated proton spectrum with different sets of parameters. Within three kinds of propagated parameters, the calculated spectrum can reproduce the AMS-02 observation. In addition, similar conclusion as $\mathrm{B} / \mathrm{C}$ ratio can be arrived at, that is, the propagated spectrum at solar system is decided by the perpendicular diffusion. 


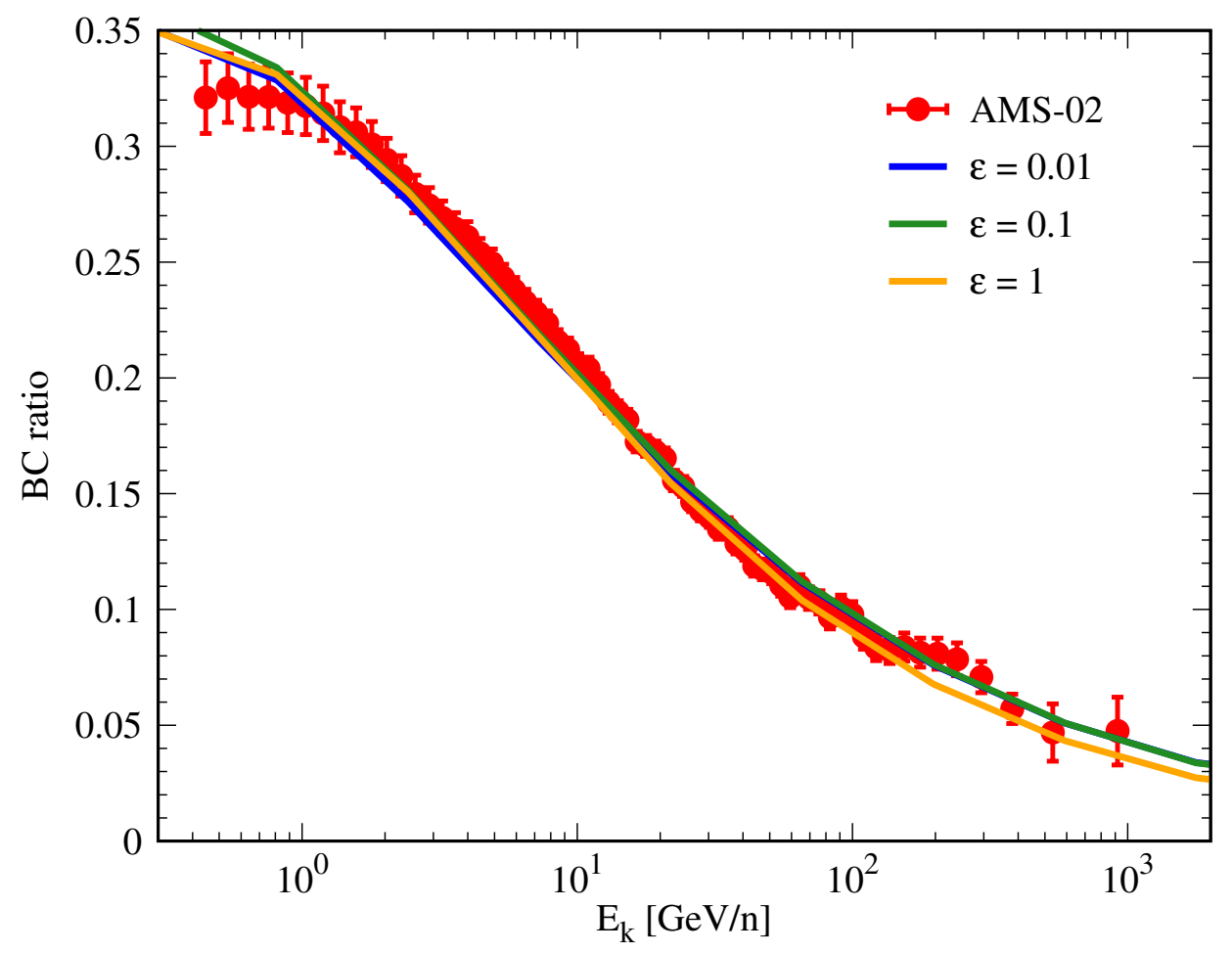

Figure 2: Fitting to $\mathrm{B} / \mathrm{C}$ ratio with different $\varepsilon$ under $3 \mathrm{D}$ anisotropic diffusion. The propagation parameters are listed in table 2. The B/C data points are taken from AMS-02 experiment [40].

\begin{tabular}{ccccccccc}
\hline \hline & $\varepsilon$ & $D_{0 \|}\left[\mathrm{cm}^{2} / \mathrm{s}\right]$ & $\delta_{\|}$ & $\delta_{\perp}$ & $\eta$ & $v_{1}$ & $\mathscr{R}_{0}[\mathrm{GV}]$ & $v_{2}$ \\
\hline Set 1 & 1.0 & $5.6 \times 10^{28}$ & 0.3 & 0.5 & 0.5 & 2.44 & 350 & 2.34 \\
Set 2 & 0.1 & $3.6 \times 10^{29}$ & 0.3 & 0.5 & 0.5 & 2.44 & 350 & 2.34 \\
Set 3 & 0.01 & $1.3 \times 10^{30}$ & 0.3 & 0.48 & 0.5 & 2.44 & 350 & 2.34 \\
\hline
\end{tabular}

Table 2: Parameters of propagation and injection spectra. The proton flux is normalized to $4.6 \times 10^{-9} \mathrm{~cm}^{-2}$ $\mathrm{sr}^{-1} \mathrm{~s}^{-1} \mathrm{MeV}^{-1}$ at $100 \mathrm{GeV}$.

\section{Summary and Discussion}

In this work, a practical pseudo source method has been proposed to numerically solve the anisotropic diffusion equation. The validity of our method is verified by comparing with previous works [33] under a toy magnetic field model. Under such situation, we further study the anisotropic diffusion by using a realistic magnetic field model. The B/C ratio and proton spectrum are calculated, both of which are consistent with AMS-02 observations.

The local regular magnetic field has been measured accurately by IBEX [42]. However such fine structure has not been accommodated in any of current magnetic field models. Accurate comparison between calculation and observed anisotropy needs further study in the future. 


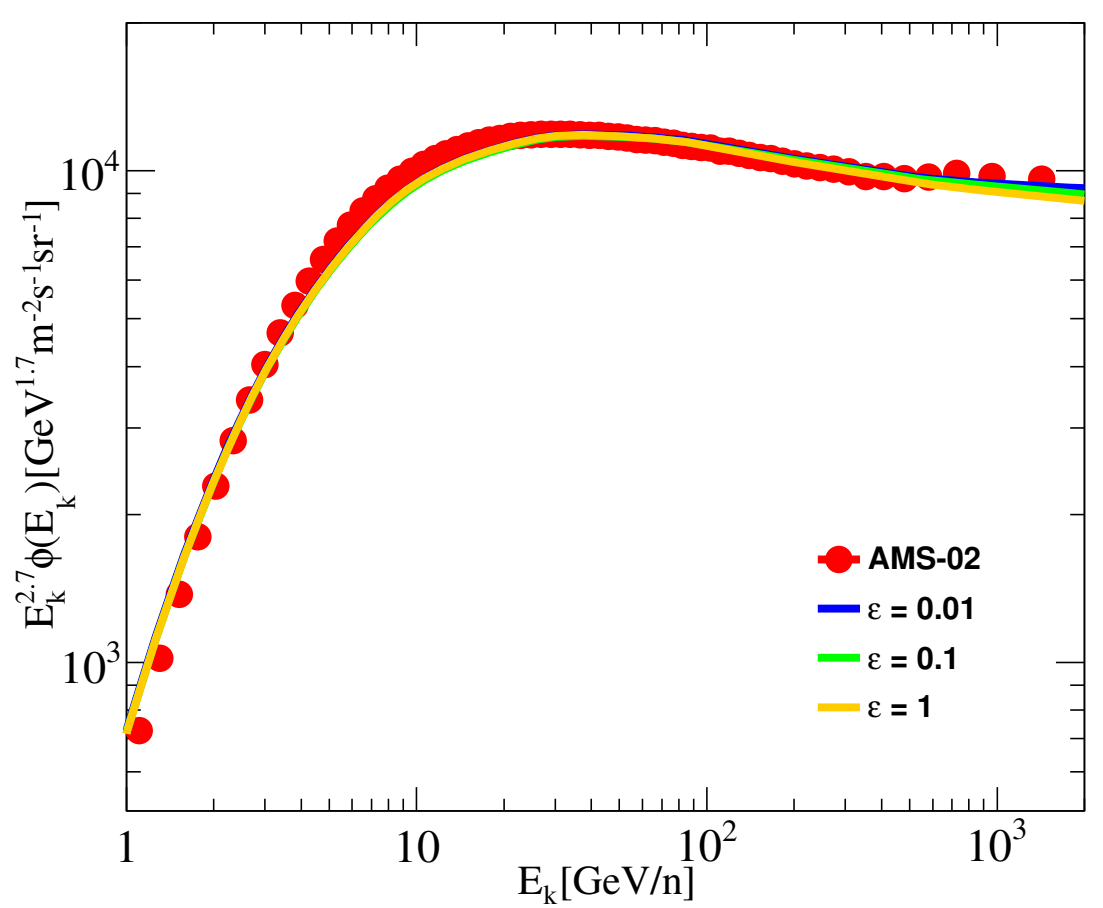

Figure 3: Calculated proton spectra with different $\varepsilon$ under 3D anisotropic diffusion. The propagation and injection parameters are listed in table 2. The proton data points are taken from AMS-02 experiment [41].

\section{Acknowledgements}

This work is supported by the National Key Research and Development Program of China (No. 2016YFA0400200, 2018YFA0404203) and Natural Sciences Foundation of China (11851303).

\section{References}

[1] Q. Yuan, S.-J. Lin, K. Fang, and X.-J. Bi. Propagation of cosmic rays in the AMS-02 era. Phys. Rev. D, 95(8):083007, April 2017.

[2] M. Amenomori, S. Ayabe, S. W. Cui, et al. Large-Scale Sidereal Anisotropy of Galactic Cosmic-Ray Intensity Observed by the Tibet Air Shower Array. ApJ, 626:L29-L32, June 2005.

[3] M. Amenomori, S. Ayabe, X. J. Bi, et al. Anisotropy and Corotation of Galactic Cosmic Rays. Science, 314:439-443, October 2006.

[4] G. Guillian, J. Hosaka, K. Ishihara, et al. Observation of the anisotropy of $10 \mathrm{TeV}$ primary cosmic ray nuclei flux with the Super-Kamiokande-I detector. Phys. Rev. D, 75(6):062003, March 2007.

[5] A. A. Abdo, B. Allen, T. Aune, et al. Discovery of Localized Regions of Excess 10-TeV Cosmic Rays. Physical Review Letters, 101(22):221101, November 2008.

[6] A. A. Abdo, B. T. Allen, T. Aune, et al. The Large-Scale Cosmic-Ray Anisotropy as Observed with Milagro. ApJ, 698:2121-2130, June 2009. 
[7] M. Amenomori, X. J. Bi, D. Chen, et al. On Temporal Variations of the Multi-TeV Cosmic Ray Anisotropy Using the Tibet III Air Shower Array. ApJ, 711:119-124, March 2010.

[8] R. Abbasi, Y. Abdou, T. Abu-Zayyad, et al. Measurement of the Anisotropy of Cosmic-ray Arrival Directions with IceCube. ApJ, 718:L194-L198, August 2010.

[9] R. Abbasi, Y. Abdou, T. Abu-Zayyad, et al. Observation of Anisotropy in the Arrival Directions of Galactic Cosmic Rays at Multiple Angular Scales with IceCube. ApJ, 740:16, October 2011.

[10] R. Abbasi, Y. Abdou, T. Abu-Zayyad, et al. Observation of Anisotropy in the Galactic Cosmic-Ray Arrival Directions at $400 \mathrm{TeV}$ with IceCube. ApJ, 746:33, February 2012.

[11] M. G. Aartsen, R. Abbasi, Y. Abdou, et al. Observation of Cosmic-Ray Anisotropy with the IceTop Air Shower Array. ApJ, 765:55, March 2013.

[12] B. Bartoli, P. Bernardini, X. J. Bi, et al. Medium scale anisotropy in the TeV cosmic ray flux observed by ARGO-YBJ. Phys. Rev. D, 88(8):082001, October 2013.

[13] A. U. Abeysekara, R. Alfaro, C. Alvarez, et al. Observation of Small-scale Anisotropy in the Arrival Direction Distribution of TeV Cosmic Rays with HAWC. ApJ, 796:108, December 2014.

[14] B. Bartoli, P. Bernardini, X. J. Bi, et al. ARGO-YBJ Observation of the Large-scale Cosmic Ray Anisotropy During the Solar Minimum between Cycles 23 and 24. ApJ, 809:90, August 2015.

[15] M. G. Aartsen, K. Abraham, M. Ackermann, et al. Anisotropy in Cosmic-Ray Arrival Directions in the Southern Hemisphere Based on Six Years of Data from the IceCube Detector. ApJ, 826:220, August 2016.

[16] M. Amenomori, X. J. Bi, D. Chen, et al. Northern Sky Galactic Cosmic Ray Anisotropy between 10 and $1000 \mathrm{TeV}$ with the Tibet Air Shower Array. ApJ, 836:153, February 2017.

[17] A. D. Erlykin and A. W. Wolfendale. The anisotropy of galactic cosmic rays as a product of stochastic supernova explosions. Astroparticle Physics, 25:183-194, April 2006.

[18] P. Blasi and E. Amato. Diffusive propagation of cosmic rays from supernova remnants in the Galaxy. II: anisotropy. J. Cosmology Astropart. Phys., 1:11, January 2012.

[19] L. G. Sveshnikova, O. N. Strelnikova, and V. S. Ptuskin. Spectrum and anisotropy of cosmic rays at TeV-PeV-energies and contribution of nearby sources. Astroparticle Physics, 50:33-46, December 2013.

[20] W. Liu, X.-J. Bi, S.-J. Lin, B.-B. Wang, and P.-F. Yin. Excesses of cosmic ray spectra from a single nearby source. Phys. Rev. D, 96(2):023006, July 2017.

[21] C. Evoli, D. Gaggero, D. Grasso, and L. Maccione. Common Solution to the Cosmic Ray Anisotropy and Gradient Problems. Physical Review Letters, 108(21):211102, May 2012.

[22] N. Tomassetti. Origin of the Cosmic-Ray Spectral Hardening. ApJ, 752:L13, June 2012.

[23] Y.-Q. Guo, Z. Tian, and C. Jin. Spatial-dependent Propagation of Cosmic Rays Results in the Spectrum of Proton, Ratios of P/P, and B/C, and Anisotropy of Nuclei. ApJ, 819:54, March 2016.

[24] N. A. Schwadron, F. C. Adams, E. R. Christian, et al. Global Anisotropies in TeV Cosmic Rays Related to the Sun's Local Galactic Environment from IBEX. Science, 343:988-990, February 2014.

[25] P. Mertsch and S. Funk. Solution to the Cosmic Ray Anisotropy Problem. Physical Review Letters, 114(2):021101, January 2015. 
[26] M. Ahlers. Deciphering the Dipole Anisotropy of Galactic Cosmic Rays. Physical Review Letters, 117(15):151103, October 2016.

[27] V. S. Berezinskii, S. V. Bulanov, V. A. Dogiel, and V. S. Ptuskin. Astrophysics of cosmic rays. Amsterdam: North-Holland, 1990, edited by Ginzburg, V.L., 1990.

[28] R. Schlickeiser. Cosmic Ray Astrophysics. 2002.

[29] A. W. Strong, I. V. Moskalenko, and V. S. Ptuskin. Cosmic-Ray Propagation and Interactions in the Galaxy. Annual Review of Nuclear and Particle Science, 57:285-327, November 2007.

[30] D. Maurin, R. Taillet, F. Donato, et al. Galactic Cosmic Ray Nuclei as a Tool for Astroparticle Physics. ArXiv Astrophysics e-prints, December 2002.

[31] G. Giacinti, M. Kachelrieß, D. V. Semikoz, and G. Sigl. Cosmic ray anisotropy as signature for the transition from galactic to extragalactic cosmic rays. J. Cosmology Astropart. Phys., 7:031, July 2012.

[32] J. Giacalone and J. R. Jokipii. The Transport of Cosmic Rays across a Turbulent Magnetic Field. ApJ, 520:204-214, July 1999.

[33] S. S. Cerri, D. Gaggero, A. Vittino, C. Evoli, and D. Grasso. A signature of anisotropic cosmic-ray transport in the gamma-ray sky. J. Cosmology Astropart. Phys., 10:019, October 2017.

[34] C. Evoli, D. Gaggero, A. Vittino, et al. Cosmic-ray propagation with DRAGON2: I. numerical solver and astrophysical ingredients. J. Cosmology Astropart. Phys., 2:015, February 2017.

[35] K. M. Ferrière. The interstellar environment of our galaxy. Reviews of Modern Physics, 73:1031-1066, October 2001.

[36] A. Fletcher, R. Beck, A. Shukurov, E. M. Berkhuijsen, and C. Horellou. Magnetic fields and spiral arms in the galaxy M51. MNRAS, 412:2396-2416, April 2011.

[37] R. Beck. Magnetic Fields in Galaxies. Space Sci. Rev., 166:215-230, May 2012.

[38] M. S. Pshirkov, P. G. Tinyakov, P. P. Kronberg, and K. J. Newton-McGee. Deriving the Global Structure of the Galactic Magnetic Field from Faraday Rotation Measures of Extragalactic Sources. ApJ, 738:192, September 2011.

[39] R. Jansson and G. R. Farrar. A New Model of the Galactic Magnetic Field. ApJ, 757:14, September 2012.

[40] M. Aguilar, L. Ali Cavasonza, G. Ambrosi, et al. Precision Measurement of the Boron to Carbon Flux Ratio in Cosmic Rays from 1.9 GV to 2.6 TV with the Alpha Magnetic Spectrometer on the International Space Station. Physical Review Letters, 117(23):231102, December 2016.

[41] M. Aguilar, D. Aisa, B. Alpat, et al. Precision Measurement of the Proton Flux in Primary Cosmic Rays from Rigidity $1 \mathrm{GV}$ to $1.8 \mathrm{TV}$ with the Alpha Magnetic Spectrometer on the International Space Station. Physical Review Letters, 114(17):171103, May 2015.

[42] D. J. McComas, F. Allegrini, P. Bochsler, et al. Global Observations of the Interstellar Interaction from the Interstellar Boundary Explorer (IBEX). Science, 326:959, November 2009. 